\title{
DIÁLOGO, ECOS Y RECOVECOS: LA COMUNICACIÓN CIENTÍFICA EN EL ÁMBITO ACADÉMICO
}

\section{DIALOGUE, ECHOES AND CRANNIES: THE SCIENTIFIC COMMUNICATION IN ACADEMIC AMBIT}

\author{
Andrés Castillo Vargas* \\ Tatiana Blanco Álvarez** \\ Esteban Montenegro Montenegro*** \\ Carlos Mata Marín**
}

RESUMEN

\begin{abstract}
El presente artículo constituye una reflexión teórica en torno a las características y la importancia de los procesos de comunicación científica, dentro del mundo académico. La comunicación de la ciencia es un proceso que los y las investigadores(as) utilizan para dar a conocer sus hallazgos, lo cual se dirige a pares académicos o públicos especializados, o a públicos más amplios y heterogéneos. La comunicación de la ciencia puede llevarse a cabo de manera directa, a cargo de quien investiga o bien, por mediación de profesionales en comunicación, quienes hacen llegar al gran público, el conocimiento que se genera en las esferas académicas. Comprender el engranaje del proceso de comunicar la ciencia es fundamental para crear estrategias efectivas que permitan su desarrollo y visualización como un objetivo propio del proceso de investigación científica.
\end{abstract}

PALABRAS CLAVE: CIENCIA * COMUNICACIÓN * PERIODISTA * INVESTIGADOR * MEDIOS DE COMUNICACIÓN * DIVULGACIÓN CIENTÍFICA

Instituto de Investigaciones Psicológicas (IIP) de la Universidad de Costa Rica (UCR).

andres.castillo@ucr.ac.cr

** Instituto de Investigaciones Psicológicas (IIP) de la Universidad de Costa Rica (UCR).

tatianamaria.blanco@ucr.ac.cr

*** Institute for Measurement, Methodology, Analysis and Policy (IMmAP), Texas Tech University. estebanmonte@gmail.com

**** Instituto de Investigaciones Psicológicas (IIP) de la Universidad de Costa Rica (UCR). carlos.matamarin@ucr.ac.cr 


\section{ABSTRACT}

This paper is a theoretical reflection on the nature and importance of the processes of scientific communication within the academic world. The communication of science is a process used by researchers to present their findings; it may be aimed at specialized academic or public peers, or wider and heterogeneous public. Communicating science can be done directly, by who investigates or by mediation of professionals in journalism, who do reach the public to the knowledge generated in academic areas. Understanding the process of communicating gear science is crucial to create effective strategies for their development and visualization as a specific objective of the process of scientific research.

KEYWORDS: SCIENCE $*$ COMMUNICATION $*$ JOURNALIST $*$ RESEARCH WORKERS $*$ MASS MEDIA * POPULARIZATION

\section{INTRODUCCIÓN}

El conocimiento científico aumenta y se fortalece en la medida en que es comunicado a los diferentes públicos que existen dentro de la sociedad, es por esta razón que puede afirmarse que "la ciencia que cuenta es la que se cuenta".

Los procesos de comunicación de la ciencia se distinguen según el público al cual se encuentren dirigidos. Se habla de difusión científica cuando la información va dirigida a grupos de pares o especialistas, $y$ de divulgación de la ciencia cuando el público al cual se dirige es diverso y heterogéneo, en ocasiones, con nulo conocimiento en la materia; a esta actividad también se le conoce como comunicación pública de la ciencia (Rivera-Tapia, 2002). Ambos procesos pueden ser desarrollados por las mismas personas que realizan las investigaciones o bien, con la mediación de profesionales en comunicación u otros mediadores, cuya tarea fundamental es transmitir la información de manera sencilla y clara a públicos cada vez más amplios y segmentados que demandan conocer acerca del quehacer científico.

El presente artículo pretende brindar un marco conceptual claro con respecto al proceso de la comunicación científica, sus ramificaciones y sus implicaciones para el desarrollo, tanto de la ciencia como de la academia. Para esto se trabajan cuatro apartados, en el primero se caracteriza el proceso de la comunicación científica y sus subtipos, en el segundo se contextualiza dicho proceso en el quehacer académico $y$ sus implicaciones, seguidamente se explica el papel de los profesionales en periodismo en el desarrollo de la comunicación de la ciencia $y$ por último, se presentan algunas reflexiones importantes en torno al tema.

Si bien existen varias actividades que conforman la llamada comunicación pública de la ciencia (educación científica, transferencia de conocimiento, entre otras), se brinda especial atención al periodismo científico, dado que los periodistas científicos poseen el conocimiento básico para mediar entre dos mundos que ocasionalmente parecen distantes, la ciencia y la sociedad; es por ello que el presente artículo desea resaltar su papel estratégico en los procesos de comunicación científica.

Se espera que las siguientes líneas permitan al personal investigador $y$ periodístico tener una comprensión más amplia del papel que les corresponde en el proceso de comunicar la ciencia, además de instigar la creación de mecanismos efectivos que faciliten la ejecución de dicho proceso, por parte de todas las personas implicadas.

\section{ENTENDIENDO LA COMUNICACIÓN DE LA CIENCIA}

La comunicación pública de la ciencia constituye el conjunto de actividades comunicativas, que el personal científico e investigador, junto a los profesionales en comunicación $y$ otros profesionales que trabajan en educación no formal de la ciencia, utilizan para transmitir los procesos, los conocimientos y los resultados de su labor académica. Este 
proceso puede encontrarse dirigido a una comunidad de especialistas, a segmentos sociales específicos o a la sociedad en general; durante las últimas décadas, el número de actividades, cursos y la cantidad de profesionales dedicados a esta temática ha aumentado considerablemente (Ecklund, James y Lincoln, 2012). Los procesos de comunicación de la ciencia se pueden llevar a cabo de distintas maneras, dependiendo - entre otras cosas - del público meta al cual se dirija.

Calvo y Calvo (2011) señalan que la comunicación de la ciencia no solo es un factor de crecimiento del propio quehacer científico, sino también una aportación para mejorar la calidad de vida de la ciudadanía y un medio para poner a disposición de muchas personas el gozo por conocer el aprovechamiento de los recursos que ofrece la naturaleza $y$ la manera en que son utilizados por la ciencia, por lo cual deben acortarse las fronteras que dividen a la ciencia de la sociedad y de otras expresiones culturales de conocimiento.

En este sentido, se pueden identificar como principales procesos de comunicación científica, la difusión y la divulgación, los cuales a su vez pueden subdividirse en otros procesos de acuerdo con los intereses del emisor y la especificidad del público; característica que particulariza aún más la labor de comunicación, al tener que tomar en consideración las peculiaridades de distintos sectores específicos (Rivera-Tapia, 2002 y Martínez, 2008).

A estos sectores específicos se les ha llamado tradicionalmente "el público" o target. Para Burns, O'Connor y Stocklmayer (2003), la definición más simple y útil de "público" es cada persona en la sociedad, con ello reconocen que "el público" es un grupo muy heterogéneo, tan multifacético e impredecible como los individuos que lo componen.

Estos autores reconocen la existencia de varias clasificaciones del "público" de acuerdo al posicionamiento teórico que se asuma, es así que puede hablarse de "público lego", refiriéndose a todas aquellas personas, incluyendo a otros científicos, que no son expertos en un determinado campo (esta nomenclatura se basa en un modelo de déficit cognitivo que ha sido fuertemente criticado por algunos sectores de los estudios sobre Ciencia, Tecnología y Sociedad (crs)).

También puede hablarse de la "comunidad científica" o "profesionales de la ciencia", que son personas que están directamente involucradas en algún aspecto de la práctica científica o de "público atento", el cual alude a las personas interesadas en ( $y$ razonablemente bien informadas acerca de) la ciencia y la actividad científica. El "público interesado" se compone de personas que están interesadas en, pero no necesariamente bien informadas de la ciencia y la tecnología.

Otros "públicos" identificados por Burns, O'Connor y Stocklmayer (2003), son la industria, la comunidad académica, el gobierno, los tecnólogos, los mediadores, los comunicadores (incluyendo comunicadores científicos, periodistas y otros miembros de los medios de comunicación), los educadores, los tomadores de decisiones (responsables políticos en el gobierno $y$ las instituciones científicas), entre otros. Asimismo, aunque Burns, O'Connor y Stocklmayer (2003), no mencionan al público con alguna discapacidad, es fundamental visualizarlo e incluirlo, al representar un segmento de la población que también posee el derecho y la necesidad de conocer $y$ acceder a información científica de primera calidad.

Desde otro posicionamiento epistemológico, Grunig (citado en Míguez, 2006) define al público:

...como un grupo de personas que: a) se enfrenta a un problema similar, b) reconoce que el problema existe y c) se organiza para hacer algo al respecto. A partir de esta definición identifica cuatro colectivos principales, los no-públicos, formados por individuos que no cumplen ninguna de estas tres características; los públicos latentes, integrados por individuos que se enfrentan a un problema similar pero no lo detectan; los públicos informados o conscientes, que están afectados por un problema similar $y$ reconocen su existencia; $y$ los públicos activos, que además de estar afectados 
por el problema y reconocerlo hacen algo al respecto... En síntesis, según Grunig, la teoría demuestra que es más probable que un público desee comunicarse si un problema le afecta, lo reconoce $y$ siente que puede hacer algo al respecto, es decir, si es un público activo (2006: 134-136).

Más allá de complejizar los procesos de comunicación de la ciencia, la especificidad en torno a la identificación de diversos públicos, se refiere a la importancia por reconocer las diferencias en el acceso al conocimiento, así como, las necesidades particulares y los intereses de cada grupo social. En conjunto, estos grupos que forman "el público", junto con sus costumbres, normas e interacciones sociales, constituyen lo que se denomina una "sociedad" (Burns et ál., 2003).

La tabla 1 retoma los aportes anteriores y esquematiza a grandes rasgos, algunas de las principales subdivisiones de los procesos de comunicación científica. Si bien dicha clasificación no cuenta del todo con un consenso internacional, constituye una posible pauta a seguir para todas aquellas personas que se encuentren interesadas en este tema.

TABLA 1

CLASIFICACIÓN DE LOS PROCESOS DE COMUNICACIÓN CIENTÍFICA

PROCESO ESPECÍFICO PÚBLICO AL CUAL SE DIRIGE SUBPROCESOS ASOCIADOS

\begin{tabular}{|c|c|c|}
\hline Difusión & $\begin{array}{l}\text { Miembros de una comunidad científica en } \\
\text { sentido amplio y/o heterogéneo. }\end{array}$ & $\begin{array}{l}\text { Diseminación: dirigida a los miembros } \\
\text { de una sola comunidad científica o de } \\
\text { especialistas. }\end{array}$ \\
\hline Divulgación & $\begin{array}{l}\text { Diversos públicos de la sociedad en } \\
\text { general. } \\
\text { De acuerdo con el contexto y el } \\
\text { posicionamiento epistemológico asumido, } \\
\text { también es conocida como popularización } \\
\text { de la ciencia, vulgarización de la ciencia, } \\
\text { comunicación pública de la ciencia, } \\
\text { cogeneración del conocimiento o } \\
\text { comprensión pública de la ciencia, entre } \\
\text { otras denominaciones. }\end{array}$ & $\begin{array}{l}\text { Periodismo científico: realizado por } \\
\text { personal periodístico, dirigido a la } \\
\text { sociedad como parte de su labor } \\
\text { informativa. } \\
\text { Educación científica: realizada en } \\
\text { espacios educativos, dirigida a estudiantes } \\
\text { y al fomento de vocaciones científicas. } \\
\text { Transferencia de conocimiento: se } \\
\text { dirige al sector productivo e industrial } \\
\text { (vinculada a procesos tecnológicos y de } \\
\text { innovación). } \\
\text { Personal científico como divulgador: } \\
\text { actividades realizadas por científicos- } \\
\text { investigadores(as) (en muchas ocasiones } \\
\text { apoyados por mediadores - periodistas, } \\
\text { gabinetes de comunicación, gestores de } \\
\text { la investigación y la comunicación, entre } \\
\text { otros-), con el objetivo de dirigirse a la } \\
\text { sociedad o a públicos específicos dentro de } \\
\text { esta, como parte de su labor de rendición } \\
\text { de cuentas y promoción de la cultura } \\
\text { científica. }\end{array}$ \\
\hline
\end{tabular}

Fuente: $\quad$ Elaboración propia con base en Rivera-Tapia (2002), Burns, O'Connor y Stocklmayer (2003), Martín y Rey (2007) y Martínez (2008). 
Considerando la clasificación anterior, los procesos de difusión y divulgación científica podrían definirse de la siguiente manera:

$\diamond \quad$ Difusión: puede comprenderse como aquellas actividades de comunicación realizadas por el personal investigador, cuyo público meta sean los miembros de una comunidad científica o de especialistas, es decir, profesionales que producen, practican $y$ validan el conocimiento científico.

$\diamond \quad$ Divulgación: se entiende como todas aquellas actividades de comunicación dirigidas a que diversos públicos posean un acceso de fácil comprensión, rápido y veraz a información científica de primera calidad. No obstante, el presente artículo brindará especial atención a una de ellas, el periodismo científico.

A partir de estas definiciones, Calvo y Calvo opinan que "los sistemas de difusión del conocimiento tienen hoy un nítido $y$ difícil objetivo, mostrar no solo el avance de las ciencias, sino sus limitaciones, y también, en ciertos casos, nuestra incapacidad para advertirlas" (2011: 19). Esta incertidumbre en la comunicación del conocimiento científico, debe ser reconocida $y$ administrada, pues cuando se habla de ciencia es imposible llegar a un nivel de total certeza, por lo cual no debe buscarse eliminar la incertidumbre sino más bien gestionarla.

Como puede notarse, "no es posible sin la ayuda de los medios conseguir que la población reciba las noticias [y comunicaciones] de ciencia y sepa entenderlas" (Calvo y Calvo, 2011: 17); sin embargo, la importancia de la comunicación científica trasciende el uso de medios y se encuentra en la relevancia social que ha adquirido con el pasar de los años, al no ser una actividad inocua $y$ hallarse atravesada por la cultura.

\section{LA COMUNICACIÓN CIENTÍFICA EN EL MUNDO ACADÉMICO}

Para Fernández y Angulo “... la ciencia ha estado inextricablemente unida a la comunicación, $y$ no puede hablarse de la primera sin la segunda" (2011: 167), dado que desde sus inicios, la difusión entre pares académicos ha sido la norma en el reconocimiento de la validez y pertinencia de los hallazgos científicos.

La comunidad científica es un mundo bastante cerrado que otorga crédito a sus miembros de acuerdo con sus propias normas y principalmente, de acuerdo con sus propios intereses. Un científico es respetable por que consigue publicar en determinadas revistas reconocidas internacionalmente, lo que a su vez le permite obtener prestigio que le facilitará el conseguir fondos para hacer nuevos trabajos e investigaciones que volverán a publicarse en otras revistas (Calvo y Calvo, 2011).

En este sentido, "la comunicación social de la ciencia es una parte integral de la propia investigación científica..." (Sanz, 2011: 65) y por ende, un proceso complementario a la creación del conocimiento.

El vínculo entre la comunicación y la ciencia ha sido "un noviazgo" colmado de recodos, que ha evolucionado en el tiempo como un binomio de gran significación en la progresividad del avance científico. El progreso científico vino a establecer como nociones fundamentales de su postura, la distinción entre lo teórico y lo observacional, al igual que la elucidación de definiciones operacionales como forma de resaltar la objetividad y rigurosidad del método científico, el cual fue catalogado como la mejor estrategia para acceder al conocimiento "verdadero" (Popper, 1934; Merton, 1973 y Echeverría, 1989).

De acuerdo con Popper (1934) y Laudan (1984), una comunidad de especialistas decide si una aportación es relevante por medio de distintos valores que aseguran la importancia de dicho descubrimiento para la comunidad, entre ellos se pueden mencionar:

$\begin{array}{ll}\diamond & \text { Ser novedosa } \\ \diamond & \text { Presentar un marco teórico de referencia } \\ \diamond & \text { Poseer rigor metodológico } \\ \diamond & \text { Ostentar consistencia interna } \\ \diamond & \text { Variedad de evidencias que permitan ser } \\ & \text { sometidas a la falsación }\end{array}$

Estas características del proceso de comunicación y difusión científica, podrían explicarse desde una visión ortodoxa de la ciencia o 
a partir del denominado enfoque interno (Merton, 1990), el cual contribuye a visibilizar la relevancia que poseen las reglas y métodos en la construcción del conocimiento y en la creación de teorías y modelos. Dicho enfoque aporta al entendimiento de la actividad científica, el carácter racional que debe distinguirle y el núcleo de rigurosidad que ha sido definido como ineludible por parte de los diversos mecanismos institucionales autorizados en la materia, los cuales resaltan la importancia de vincular la ciencia a las esferas académicas, al ser estas últimas espacios en los cuales se gestiona gran parte del conocimiento.

No obstante, durante el siglo pasado existió una polémica discusión en torno a la diferenciación de los valores fundamentales para reconocer $y$ difundir la relevancia de un aporte científico, identificándose valores epistémicos, implicados en la producción directa del conocimiento - considerados puntos clave de la racionalidad- $y$ valores no epistémicos - valores culturales y sociales - referidos al contexto en el cual se desarrolla la actividad científica, los cuales podrían ubicarse en lo que Merton (1973 y 1990) denomina enfoque externo de la ciencia.

En la primera línea de valores, Kuhn (1977) distinguió cinco características epistémicas de gran importancia que debían caracterizar a la ciencia: precisión, coherencia (no entrar en contradicciones teóricas), amplitud de alcance, simplicidad (permitir ser representada por modelos) y fecundidad (dar pie a nuevas investigaciones). Mientras que en la segunda línea, el enfoque externo ayudó a identificar la relación de la ciencia con la sociedad, vinculando el análisis de las instituciones científicas a la historia, la cultura $y$ a diversos valores ajenos a lo estrictamente metódico y más cercanos a lo social. Este enfoque propone la contextualización de la producción científica al reconocer la característica de "control" e influencia que ejerce la ciencia entre los miembros de una comunidad y viceversa (Merton, 1990 y Haack, 1996). Además, el brindar una visión más sociológica del mundo científico, ha motivado el análisis y el cuestionamiento público de las visiones fragmentadas presentes tradicionalmente en la institucionalidad científica, como por ejemplo, la crítica feminista a los sesgos de género en la ciencia y la necesidad de comunicar los resultados científicos a públicos diversos.

Dicha discusión axiológica prosiguió con el tiempo, ya que Longino (1990) planteó posteriormente un debate similar al diferenciar entre valores constitutivos (adecuación empírica, simplicidad y poder explicativo) y valores contextuales en la investigación científica, los cuales incluían la importancia de la comunicación no solo dirigida a la comunidad de personas expertas, sino también al "vulgo", destacándose así la progresividad de la ciencia al pasar de una visión esencialista y triunfalista a una posición más inclusiva que reconoce la influencia de la sociedad, la cultura y la historia en la generación del conocimiento.

Esta postura respetuosa e inclusiva de la ética social ha favorecido a que actualmente se transcienda la relación difusión-ciencia, promoviendo de manera cada vez más creciente, la relación divulgación-ciencia como una forma de entablar nuevos puntos de encuentro entre el mundo científico, la tecnología y la sociedad.

Si bien, es fundamental comprender que la difusión de la ciencia es una actividad compleja en sí misma y constitutiva del proceso de investigación, para enriquecer su debate y discusión, es necesario que el personal investigador reconozca, reflexione y participe de nuevas alternativas de comunicación, que le brinden no solo reconocimiento en la comunidad de especialistas sino también reconocimiento y valía dentro del entramado social, el cual indudablemente contribuirá a la progresividad del conocimiento científico.

Precisamente, las universidades públicas están llamadas a desarrollar con especial ahínco dicha labor al ser instituciones financiadas por la ciudadanía; las cuales deben buscar no solo el posicionamiento social, sino también, la rendición de cuentas frente a la opinión pública de los fondos asignados. Los procesos de comunicación científica no solo deben buscar la difusión o divulgación de resultados, sino también propiciar el interés $y$ la participación de la ciudadanía en los procesos de gestión del conocimiento, sus aplicaciones, alcances, riesgos e 
incertidumbres, fomentando eventualmente la alfabetización en temas científico-tecnológicos, pero principalmente, promoviendo la adquisición de una sólida cultura científica.

De esta manera, el compromiso social de comunicar la ciencia se encuentra vinculado también al establecimiento de procesos de transparencia y ética en la investigación académica, que resalten la corresponsabilidad social del personal científico en el mejoramiento de las necesidades humanas.

\section{PERIODISMO CIENTÍFICO: UN MEDIO PARA COMUNICAR LA CIENCIA}

Aunque cada vez es más notorio que profesionales de otras disciplinas están escribiendo para medios masivos, tanto en género informativo como interpretativo ${ }^{1}$; el periodismo científico puede conceptualizarse tradicionalmente como todas aquellas actividades de comunicación de información científica, realizadas por personal periodístico, que se encuentran dirigidas a la sociedad como parte de su labor informativa (Martín y Rey, 2007; Martínez, 2008). Entre las actividades más comunes que realizan los periodistas científicos se encuentran: las entrevistas, los reportajes y las noticias.

Aunque en la literatura se pueden hallar un sin número de géneros o clasificaciones periodísticas, Fernández y Angulo (2011) recomiendan su agrupación en tres grandes rubros, géneros informativos, explicativos y de opinión. Cada uno de ellos posee características diferenciales, las cuales se señalan a continuación:

$1 \quad$ Es importante mencionar que a esta vasta $y$ diversa cantidad de profesionales se les ha denominado divulgadores de la ciencia y no periodistas científicos, debido a que los conocimientos que poseen en torno a los procesos de comunicación son de carácter empírico o no formal, o en su defecto, su formación base no es la de periodista. Lo cual no minimiza ni resta importancia a su labor, todo lo contrario. No obstante, tampoco es lícito equipar dicho conocimiento a las destrezas formales que poseen los profesionales que han completado la carrera de periodismo, con especialidad o énfasis en periodismo científico. $\diamond \quad$ Géneros informativos (narran hechos): la noticia es su mayor representante, se caracteriza por su actualidad, singularidad y proximidad. De acuerdo con Goñi (2011), el primer párrafo o lead de una noticia debe responder a la teoría de las cinco "W" (who, what, where, when and why), es decir: quién, qué, donde, cuándo $y$ por qué.

$\diamond \quad$ Géneros explicativos (contextualizan hechos): intentan contextualizar un contenido de actualidad, el reportaje es el ejemplo más fiel de este tipo de género, el cual ofrece información detallada $y$ completa sobre un tema concreto, buscando contrastar diferentes fuentes e inter locutores.

$\diamond \quad$ Géneros de opinión u opinativos (brindan opiniones): las columnas de opinión o las de editorial (opinión de un medio), al igual que el artículo periodístico, son un ejemplo de este tipo de género en el cual se usa tradicionalmente la primera persona. El lenguaje suele ser literario, admitiendo el cinismo y el sarcasmo que son poco utilizados en otros géneros.

De acuerdo con De Semir (2000), la cantidad de noticias científicas que se localizan en los encabezados de prensa ha crecido dramáticamente, de la misma manera en la que el rol de la ciencia en la sociedad va en incremento. Situación que evidencia cómo la relación entre el mundo de la ciencia y los medios de comunicación es inevitable y principalmente, positiva, aunque también puede resultar algo complicada.

Las noticias son un producto de la sociedad y como todos los productos, son preparadas por profesionales. El periodista debe convertir la información de un recurso especializado, a algo que pueda ser comprendido por una audiencia más general que carece de conocimiento previo sobre esta información. Este mundo de la comunicación establece sus propias normas, imágenes y su propio lenguaje al momento de comunicar contenidos científicos complejos, para ello hacen uso de recursos tan diversos como las metáforas u otras 
figuras literarias con el fin hacer accesible la información científica a un público heterogéneo (De Semir, 2000).

Si bien, el uso de metáforas puede contribuir a que la ciencia no sea percibida como una "caja cerrada ininteligible" carente de características que la vuelvan interesante al gran público, se debe evitar caer en el error de amenizar la actividad científica buscando hacerla divertida por medio de la trivialización, la frivolidad, la superficialidad o las distorsiones ideológicas (Palma, 2013), ya que el uso de estos recursos lo que promueve es una visión degradada y distorsionada de los descubrimientos científicos.

La tabla 2 muestra algunos de los problemas más frecuentes que se presentan en las noticias sobre ciencia, los cuales deben ser evitados con miras a brindar una idea adecuada de la investigación científica.

TABLA 2

PROBLEMAS FRECUENTES EN EL PERIODISMO AL MOMENTO DE COMUNICAR NOTICIAS CIENTÍFICAS

\section{PROBLEMA}

EJEMPLOS

Empleo burdo de la ciencia en las noticias referidas a las áreas biológicas o biomédicas.

Tendencia a eliminar las diferencias entre animales $y$ humanos de un modo exagerado o ilegítimo epistemológica y conceptualmente, lo cual conlleva a la antropomorfización de los animales y/o a la zoologización de los seres humanos. genéticos". cerebro artificial". ser corruptas". hormigas".
La Nación (14/04/2008) "Una tienda del Soho ofrece tests

El País (02/03/2009) "Una clínica de EEUu ofrece niños a la carta". Clarín (08/04/2009) "Creen que en diez años puede existir un

BBC Ciencia (06/06/2008) "No es pereza, son sus genes".

Clarín (16/03/2008) "Descubren que las hormigas también pueden

Página 12 (03/01/2009) "Biología y división del trabajo: hormigas comunistas. De especialistas y diletantes".

La Nación (06/02/2009) "Orugas impostoras engañan a las

Clarín (15/07/2009) "Los tiburones blancos atacan como si fueran asesinos seriales".

The New York Times (05/10/2013) "Dogs Are People, Too" [Los perros también son personas].

Uso de correlaciones caprichosas o espurias como si fueran relaciones causales.

Clarín (11/09/2007) "Dicen que el tamaño de los dedos influye en la inclinación de los chicos". Clarín (04/03/2008) "Tener un gato como mascota reduciría el riesgo de infarto. En quienes no viven con estos animales aumentaría un $40 \%$ las probabilidades de morir por esa causa".

Presentación de investigaciones triviales como si fueran grandes descubrimientos de la ciencia.

Infobae (11/12/2008) "Científicos calcularon la velocidad exacta a la que sale el corcho del champán".

Noticias Repretel (26/09/2013) "Mujeres con trasero grande son más inteligentes y viven más según un estudio".

La Nación (06/10/2008) "Stonehenge podría haber sido... un spa". El Mundo (04/07/2012) "¿Qué supondría el hallazgo de la 'partícula de Dios'?".

La Nación (24/06/2008) "Hallan precisiones astronómicas en la poesía de Homero. La Odisea, a la luz de la ciencia”.

El Mundo (14/3/2013) "cBs News ubicó la nacionalidad del papa Francisco en Colombia”.

Fuente: elaboración propia con base en Palma, 2013. 
Todos los ejemplos expuestos con anterioridad, evidencian cómo en ocasiones el periodismo científico ofrece una imagen descontextualizada, falsa y estereotipada de la ciencia. La práctica científica aparece como una tarea ingenua y ahistórica, minimizando la importancia del debate y los conflictos ideológicos y paradigmáticos en el avance del conocimiento, promoviendo asociaciones religiosas o morales que acercan la ciencia a esferas míticas, superficiales y pseudocientíficas.

De acuerdo con Palma (2013), se debe analizar cuidadosamente qué porcentaje de los problemas que ocurren en los medios de comunicación al reportar noticias sobre ciencia se deben, por un lado, al personal científico y por el otro, a los periodistas. Para dicho investigador:

...las metáforas científicas no solo son un recurso didáctico, retórico o heurístico válido para comunicar la ciencia, son fundamentalmente un recurso cognoscitivo $y$ explicativo insustituible. En este sentido, la metáfora no opera solamente como una explicación alternativa para aquellos que no saben sobre algún tema [sino también como un recurso de mediación pedagógica que facilita el entendimiento de contenidos complejos; el problema se presenta cuando el uso de dichas metáforas es poco prudente y sensacionalista] (Palma, 2013: 21).

Por otra parte, una consecuencia bastante habitual de problemas como la antropomorfización del mundo animal en las noticias sobre ciencia, es la inclusión de un discurso moralizador que no solo es aplicado de manera ilegítima al mundo animal, sino que además responde a pautas de una moral tradicional, plagada de prejuicios e ideas falsas acerca de las relaciones humanas y de la ciencia (Palma, 2013).

Este discurso basado en la moral tradicional, permea en muchas ocasiones los procesos de comunicación científica realizados por los medios de comunicación, es así que abundan en la ciencia las metáforas de tipo religioso:

No hace mucho se realizó una gran operación mediática a partir de la puesta en funcionamiento del Gran Colisionador de Hadrones del cers (Organización Europea para la Investigación Nuclear) al que se denominó "la máquina de Dios". A su vez, entre sus tareas más relevantes en términos teóricos estaba encontrar la "partícula de Dios" o "Bosón de Higgs", una partícula elemental hipotética (Palma, 2013: 24).

Todas estas metáforas en torno al Bosón de Higgs acercaban más dicho descubrimiento a lo divino que a lo científico, contribuyendo a crear una visión errónea de la investigación en partículas nucleares:

Tampoco hay que ignorar la desconfianza que los científicos tienen en la divulgación científica en general, así como también el menosprecio que las comunidades científicas sienten por el trabajo de divulgación, descalificación que se hace patente en los sistemas de evaluación por pares que le otorgan poco o ningún valor, o incluso son evaluadas negativamente (Palma, 2013: 27).

En este punto, resulta relevante comprender que la realidad científica y la realidad en los medios no son el mismo asunto. Muchos descubrimientos científicos tienen aplicaciones prácticas que ayudan a validarlos, pero algunos otros muestran controversias e incertidumbres que indican nuevas direcciones de investigación sin una aplicación práctica inmediata (De Semir, 2000).

En este sentido, es fundamental mostrar la importancia de divulgar las controversias científicas de una manera seria y responsable, planteando ante el gran público la importancia del debate y la discusión en la génesis del conocimiento, aceptando la eventual existencia de controversias en la búsqueda de nuevos saberes, pues gracias a estas se posibilitan nuevas investigaciones y la aparición de nuevos modelos y teorías científicas.

En conclusión, la comunicación de noticias científicas a través de los medios de comunicación no es una tarea fácil. Sin embargo, periodistas e investigadores(as) se están dando 
cuenta que sus profesiones se encuentran cada vez más entrelazadas, por lo cual están explorando nuevas formas de trabajo en conjunto, en donde la vinculación entre el mundo de la ciencia y los medios de comunicación sea el primer paso en el difícil proceso de transformar el discurso científico en conocimiento público.

\section{REFLEXIONES FINALES}

Como se ha mencionado en acápites anteriores, la relación entre la academia y los medios, es fundamental en el fortalecimiento de la investigación científica, no solo porque contribuye a su reconocimiento, sino también por que promueve la cultura científico-tecnológica de la ciudadanía.

Considerando esta premisa, hay que reconocer que las universidades están llamadas a ser protagonistas del diálogo con el público; al constituirse en una pieza indispensable para la formación de ciudadanos activos y eficaces frente a los avances científicos y tecnológicos.

Reflexionando en torno a esta idea, Chiappe y Fazio consideran que las actividades para la promoción de la cultura científica han aumentado en los últimos años; sin embargo: "muchas de estas actividades se concentran en la promoción de la cultura vinculada a las ciencias exactas $y$ naturales $y$ a aplicaciones tecnológicas, pero son muy pocas las que se desarrollan en torno a las ciencias sociales $y$ las humanidades" (2011: 358).

Esta situación podría originar una visión fragmentada de la ciencia, pues incurre en el error de asociar lo "científico" de forma exclusiva a las ciencias duras o exactas, lo cual debe llevar a las instituciones académicas a identificar nuevas estrategias de comunicación para acercar a las ciencias sociales y las humanidades al público, evitando así su exclusión en la formación de la cultura científica ciudadana.

Desde este posicionamiento epistemológico, la producción académica permanente en todas las áreas del conocimiento científico permite alcanzar niveles de excelencia en la formación de profesionales, los cuales a su vez, pueden desempeñarse como eventuales difusores $y$ agentes de cambio para la sociedad en general (Universidad de Costa Rica, 2013).
La Universidad de Costa Rica (ucR) es justamente una universidad pública que toma en consideración las premisas esbozadas con anterioridad. Fue creada el 26 de agosto de 1940 y es la institución de educación superior pública más antigua de Costa Rica, su población estudiantil asciende a los 35000 estudiantes y posee más de cincuenta unidades de investigación; esto conlleva a que produzca aproximadamente el 80\% de las publicaciones indexadas internacionalmente del país y cerca del 25\% de América Central (Parral y Vindas, 2009).

Para la ucR, las actividades de investigación constituyen una de sus tareas básicas, junto con la docencia y la acción social. Es de esta manera, que en la mayoría de escuelas o facultades funcionan programas de investigación de significativa productividad, además de existir diversos centros e institutos de investigación, los cuales son un ejemplo de cómola comunicación y la investigación académica dentro de la ucR, se encuentran al servicio una de la otra desde la génesis misma de la actividad científica, siendo procesos que han evolucionado de manera conjunta.

El reconocimiento de la función científica y social de las universidades, ha procurado ampliar el espectro comunicativo de la ciencia, destacando no solo la importancia de la difusión como mecanismo de reconocimiento $y$ validación, sino también la necesidad de efectuar procesos de divulgación como una forma de popularizar y acercar la ciencia a la sociedad.

Comunicar la ciencia es un proceso complejo, en el cual no siempre los actores involucrados persiguen el mismo fin. Es por esta razón, que resulta de vital importancia entablar procesos de mediación entre el personal investigador $y$ los periodistas para que en conjunto puedan desarrollar eficaces procesos de comunicación. No obstante, antes de efectuar tan insigne labor, es fundamental acercarse a la percepción que los científicos-investigadores(as) poseen de los periodistas con los cuales suelen tratar, y acercarse a sus actitudes y prácticas respecto a dicha relación, tarea que en sí misma implica diversos derroteros. 
Mejorar las relaciones entre investigadores(as) y periodistas es indiscutiblemente una manera de mejorar la confianza y derribar los muros del desconocimiento en torno a ambas profesiones, el fortalecimiento de esta relación favorecería el establecimiento de nortes claros en lo que compete a la rendición de cuentas a la sociedad, por parte de aquellos profesionales a los cuales se les encomienda crear y comunicar el conocimiento.

Es importante que quienes investigan no piensen en su tarea como "un cántaro que chorrea" conocimiento, muy al contrario, deben pensarse a sí mismos como un eslabón más en la cadena de producción científica, que no existiría ni tendría razón de ser sin la participación de los diferentes públicos que reciben y retroalimentan su quehacer. Comprendiendo a su vez que para lograr esta tarea, deben hacer uso de todos los recursos a su disposición, siendo el personal periodístico un aliado primordial en esta labor, por el gran aporte que realizan en la divulgación del conocimiento.

Finalmente, es fundamental el desarrollo de estrategias de gestión de la comunicación que respondan realmente a las necesidades del público al cual van dirigidas. En este sentido, la capacitación en dicho tema, es el primer paso ineludible $y$ primordial para construir relaciones efectivas entre investigadores(as), periodistas y públicos. En la medida en que cada actor conozca las necesidades, competencias, límites, habilidades y fortalezas que poseen, se podrá construir un diálogo sincero, en el cual no existan ecos ni recovecos que entorpezcan el proceso de comunicar la ciencia.

\section{BIBLIOGRAFÍA}

LIBROS

Calvo, Manuel y Calvo, Antonio. "De la divulgación científica a la ciencia mediática". Periodismo y divulgación científica: tendencias en el ámbito iberoamericano. Carolina Moreno (ed.). Madrid, España. Editorial Biblioteca Nueva, sL. 2011: 15-39.

Chiappe, Dolores y Fazio, María Eugenia.

"La organización de actividades para promover la cultura científica". Periodismo y divulgación científica: tendencias en el ámbito iberoamericano. Carolina Moreno (ed.). Madrid, España. Editorial Biblioteca Nueva, sL. 2011: 346-376.

Echeverría, Javier. Introducción a la metodología de la ciencia. Barcelona, España: Hurope, 1989.

Fernández, Ignacio y Angulo, Eugenia. "El lenguaje y los formatos en la comunicación de la ciencia”. Periodismo y divulgación científica: tendencias en el ámbito iberoamericano. Carolina Moreno (ed.). Madrid, España. Editorial Biblioteca Nueva, st. 2011:166-189.

Goñi, Ainhoa. "Gestión de la información científica y técnica en las instituciones públicas". Periodismo y divulgación científica: tendencias en el ámbito iberoamericano. Carolina Moreno (ed.). Madrid, España. Editorial Biblioteca Nueva, st. 2011: 310-345

Haack, Susan. "Science as social-yes and no". Feminism, Science, and the Philosophy of Science. Lynn Nelson y Jack Nelson (eds.). London, Inglaterra. Kluwer Academic Publishers, 1996: 79-93.

Kuhn, Thomas. The essential tension. Chicago, Eeuv: University of Chicago, 1977.

Laudan, Larry. Science and values: the aim of science and their role in the scientific debate. Pittsburgh series in the philosophy and history of science. Berkeley, Eeuv: University of California Press, 1984.

Longino, Helen. Science as social knowledge: values and objectivity in scientific inquiry. Princeton, eevu: Princeton University Press, 1990.

Martín, María José y Rey, Jesús. Papel de los científicos en la comunicación de la ciencia y la tecnología a la sociedad: actitudes, aptitudes e implicación. Madrid, España: Comunidad de Madrid y csic, 2007. [Disigal]. En: <http://digital.csic.es/ bitstream/10261/1616/1/30_Papel.pdf> [consultado el 10 de julio de 2014]. 
Merton, Robert. La sociología de la ciencia. Madrid, España: Alianza editorial, 1973.

Merton, Robert. "sтs: foreshadowing of an evolving research program in the sociology of science." Puritanism and the rise of modern science: the Merton thesis. Bernard Cohen (ed.). New Brunswick and London. Rutgers University Press, 1990: 334-371.

Popper, Karl. La lógica de la investigación científica. Madrid, España: Tecnos, 1934.

Sanz, Noemí. "La perspectiva cts en el estudio y reflexión sobre la comunicación social de la ciencia y la tecnología." Periodismo y divulgación científica: tendencias en el ámbito iberoamericano. Carolina Moreno (ed.). Madrid, España. Editorial Biblioteca Nueva, sL. 2011: 40-74.

\section{PUBLICACIONES PERIÓDICAS}

Burns, Terry; O'Connor, John y Stocklmayer, Susan. "Science communication: a contemporary definition". Public Understanding of Science 12. California, Eeuu. Sage Journals, 2003: 183-202.

De Semir, Vladimir. "Scientific journalism: problems and perspectives". International Microbiology 3. Barcelona, España. University Pompeu Fabra, 2000: 125-128.

Ecklund, Elaine; James, Sarah y Lincoln, Anne. "How academic Biologists and Physicists view science outreach". PLOS ONE 7(5). California, Eeuu. 2012: 1-5.

Martínez, Manuel. "La responsabilidad del investigador en la divulgación de la ciencia”. Revista de Divulgación Científica y Tecnológica de la Universidad Veracruzana 21(1). México. Universidad Veracruzana, 2008: 19-24.

Míguez, María Isabel. "Situational theory of publics: new contributions since the nineties". Communication \& Society 19(2). Pamplona, España. Universidad de Navarra, 2006: 133-162.

Palma, Héctor. "Algunos tópicos críticos acerca del periodismo científico en grandes medios gráficos. El rey sigue desnudo". Revista cTS 23 (8). Buenos Aires, Argentina. Universidad Nacional de San Martín, 2013: 13-30.

Rivera-Tapia, José. "Ciencia y divulgación". Revista Biomédica 13(2). Yucatán, México. Universidad Autónoma de Yucatán, 2002: 152-153.

\section{TEXTOS ELECTRÓNICOS}

Universidad de Costa Rica. "La ucr en breve." En: <http://www.ucr.ac.cr/acerca-u/u-enbreve/> [consultado el 20 de agosto 20 de 2013].

OTROS

Parral, César y Manrique Vindas. "Comunicación de la ciencia en la Universidad de Costa Rica." I Foro Iberoamericano de Comunicación y Divulgación. Campinas, Brasil, 2009.

Fecha de ingreso: 15/09/2014 Fecha de aprobación: 20/01/2015 